\title{
Increasing the Diversity of Crops That Can Be Grown in Urban and Vertical Farms ${ }^{\dagger}$
}

\author{
Cathryn A. O'Sullivan *, Graham D. Bonnett, C. Lynne McIntyre, Ian B. Dry and \\ Lekha Sreekantan \\ CSIRO Agriculture and Food, St Lucia, Brisbane 4067, QLD, Australia; Graham.Bonnett@csiro.au (G.D.B.); \\ Lynne.Mcintyre@csiro.au (C.L.M.); Ian.Dry@csiro.au (I.B.D.); Lekha.Sreekantan@csiro.au (L.S.) \\ * Correspondence: cathryn.osullivan@csiro.au \\ + Presented at the third International Tropical Agriculture Conference (TROPAG 2019), Brisbane, Australia, \\ 11-13 November 2019.
}

Published: 5 January 2020

\begin{abstract}
The FAO estimates that more than 800 million people engage in urban agriculture producing more than $15 \%$ of the world's food. Recently, there has been a resurgence of interest in urban agriculture in many wealthy, developed cities, with new technology and agro-architecture being employed to grow food in cities at commercial scale. This has been accompanied by an increase in media coverage. Big claims are being made, including that urban agriculture can help solve food security for growing urban populations, decrease greenhouse emissions, 'climate proof' farms, and provide chemical free food with no risk of pests and diseases. Many of these claims need to be rigorously tested to ensure that sound investments can be made in enterprises that are financially viable and capable of delivering on claims of social and environmental benefits. Traditionally, agricultural researchers have provided biological, chemical, physical, economic and social research help broad-acre and horticulture farming increases productivity and decrease risk. Urban agriculture needs similar support as the industry grows and develops around the world. There are opportunities to improve crop yields and quality by pairing advancements in environmental controls, phenomics and automation with breeding efforts to adapt traits for architecture, development and quality (taste and nutrition) allowing a more diverse set of crops to be grown in controlled-environment farms. CSIRO is looking to apply our establish capability and skills to support the urban and vertical farming industry to contribute to the nutrition of city dwellers as urban populations continue to rise.
\end{abstract}

Keywords: indoor agriculture; vertical farming; controlled environment farming; protected cropping; horticulture 